\title{
Design of a Latent Heat Energy Storage System Coupled with a Domestic Hot Water Solar Thermal System
}

\author{
Robynne Murray $^{1,3}$, Louis Desgrosseilliers ${ }^{1,2,3}$, Jeremy Stewart ${ }^{1}$, Nick Osbourne ${ }^{1}$, \\ Gina Marin ${ }^{1}$, Alex Safatli ${ }^{2}$, Dominic Groulx ${ }^{1,3^{*}}$, Mary Anne White ${ }^{2,3}$ \\ ${ }^{1}$ Department of Mechanical Engineering, Dalhousie University, Halifax, Canada \\ ${ }^{2}$ Department of Chemistry, Dalhousie University, Halifax, Canada \\ ${ }^{3}$ Institute for Research in Materials, Dalhousie University, Halifax, Canada \\ * Corresponding author. Tel: +1 902 494-8835, Fax: +1 902 423-6711, E-mail: dominic.groulx@dal.ca
}

\begin{abstract}
Solar domestic hot water (SDHW) can be used to reduce energy bills and greenhouse gas emissions associated with heating domestic water. However, one of the most significant barriers to further deployment of solar thermal applications is the space and weight required for storage of the energy collected. Phase change materials (PCMs) are advantageous for daily energy storage with SDHW due to their high storage density and isothermal operation during phase transitions, and would overcome these obstacles.
\end{abstract}

The aim of this paper is to outline the initial steps in the development of a SDHW energy storage system using PCMs, with emphasis on the numerical and experimental studies used to access the phase change and thermal behaviour of the selected PCM. Lauric acid was selected as the PCM based on the melting temperature range which was targeted by studying solar data from an existing solar hot water system in Halifax, Nova Scotia, Canada. Due to the low thermal conductivity of PCMs, additional work is required to develop and validate a design to enhance heat transfer to the storage material using fins. The selected design will be built and installed in an existing large scale solar thermal system on an apartment building in Halifax. The system will be instrumented in order to acquire continuous data (temperatures, flow rates, pressures, etc.) to fully characterize the system.

Keywords: Latent heat storage, Solar domestic hot water, Phase change materials, Heat transfer enhancement

\section{Introduction}

Solar thermal energy for domestic hot water heating is one of the most cost effective and efficient areas of alternative energy exploitation [1]. The use of phase change materials (PCMs) in latent heat energy storage systems (LHESS) can reduce the volume and weight of storage due to their high storage density, and overcome major obstacles in the further deployment of solar thermal energy [1]. LHESS have high energy densities compared with sensible heat storage systems [2], and have been shown to store up to 14 times more heat per unit volume than sensible heat storage materials [3]. Fig. 1 shows a simple schematic of a SDHW system with PCM energy storage.

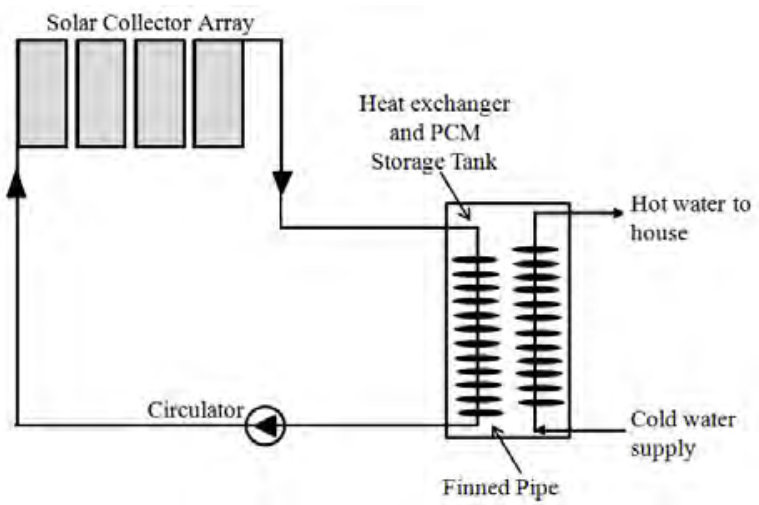

Fig. 1. Schematic of a LHESS for SDHW. 
Energy storage using PCMs in combination with solar collectors has been studied mathematically [4] and experimentally [5] and shown to be advantageous. However, missing from previous works is a working prototype of a SDHW system for a large scale application [6].

This paper presents a phase change heat transfer study performed using a PCM, lauric acid, in a small-scale experimental LHESS using fins to enhance the overall heat transfer process. A numerical model was also created and its results are compared and validated with experimental results. Results of this study are to be used in the design of a SDHW system, with the numerical model to be used further in design optimization, mainly for fin configurations, of the LHESS. The resulting LHESS design will be built and installed in an existing large scale solar thermal system on an apartment building in Halifax by Scotian Windfield Inc.

\section{Phase Change Material Selection}

The PCM is selected based on its phase change temperature range and the operating temperatures of the SDHW system. A melting temperature range of 42 to $48^{\circ} \mathrm{C}$ and solidification temperature range of 35 to $40^{\circ} \mathrm{C}$ were targeted by studying the solar data from an existing SDHW system in Halifax, Canada. Several PCMs were considered based on their appropriate melting temperatures, low toxicities, and cost. The most promising materials were tested using a differential scanning calorimeter (DSC) to study their melting and solidification temperature ranges. Salt hydrates (e.g. Glauber's salt and sodium acetate) tested in the DSC showed significant supercooling, which is a common and undesirable phenomenon for these materials [7].

The DSC curve for lauric acid (dodecanoic acid; $\mathrm{CH}_{3}\left(\mathrm{CH}_{2}\right)_{10} \mathrm{COOH}$; crude $[<80 \%$ pure], Fisher Scientific), presented in Fig. 2, shows a melting temperature range of 43.3 to $45.7{ }^{\circ} \mathrm{C}$ and solidification temperature range of 38.8 to $35^{\circ} \mathrm{C}$. The DSC curve for this lower purity sample compared well with literature curves for pure lauric acid [8]. Other fatty acids that were tested had either incompatible phase transition regions or toxicities and cost that were undesirable.

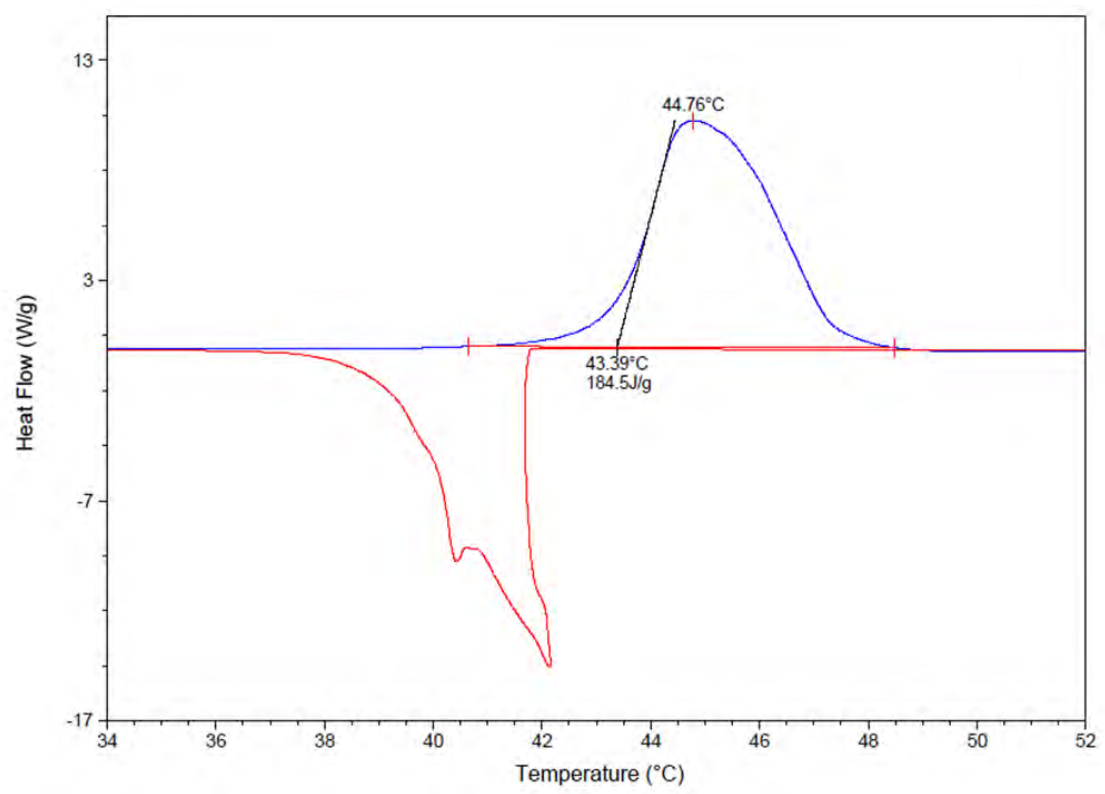

Fig. 2. DSC Curve for Lauric Acid (80\% purity) measured at $10 \mathrm{~K} / \mathrm{min}$. 
Lauric acid was selected based on its melting temperature range, high heat of fusion, minimal supercooling and safety. The material properties are displayed in Table 1.

Table 1. Thermal and Physical Properties of Lauric Acid [8,9].

\begin{tabular}{lc}
\hline Molecular Weight & $200.31(\mathrm{~kg} / \mathrm{kmol})$ \\
\hline Density of Powder at $20^{\circ} \mathrm{C} /$ Liquid at $45^{\circ} \mathrm{C}^{\S}$ & $869 / 873\left(\mathrm{~kg} / \mathrm{m}^{3}\right)$ \\
\hline Fusion Temperature & $42\left({ }^{\circ} \mathrm{C}\right)$ \\
\hline Latent Heat of Fusion & $182(\mathrm{~kJ} / \mathrm{kg})$ \\
\hline Heat Capacities Solid/Liquid ${ }^{\dagger}$ & $2.4 / 2.0(\mathrm{~kJ} / \mathrm{kg} \cdot \mathrm{K})$ \\
\hline Thermal Conductivities Solid/Liquid $^{\dagger}$ & $0.150^{*} / 0.148(\mathrm{~W} / \mathrm{m} \cdot \mathrm{K})$ \\
\hline Viscosity $^{\dagger}$ & $0.008(\mathrm{~Pa} \cdot \mathrm{s})$ \\
\hline \multicolumn{2}{c}{${ }^{*}$ Value obtained from present experiments. } \\
${ }^{\dagger}$ Nominal properties calculated near the melting point. \\
${ }^{\circledR}$ Density used in the numerical model presented $=880 \mathrm{~kg} / \mathrm{m}^{3}$.
\end{tabular}

To insure stable properties after many melting/solidification cycles, lauric acid was thermally cycled from 37 to $63{ }^{\circ} \mathrm{C}$. A fter 800 cycles, there were no obvi ous signs of degradation. Lauric acid is also safe to use in conjunction with a SDHW system because it is a food grade substance and only a mild irritant [9].

\section{Experimental Setup}

The experimental setup used to study the melting and solidification behavior of lauric acid in a cylindrical container with horizontal copper fins is shown schematically in Fig. 3a. A Solidworks 3D rendering of the container is shown in Fig. 3b. Eight type $\mathrm{T}$ probe thermocouples are connected to a National Instruments 16-channel thermocouple module (NI9213) CompactDAQ data acquisition system. Temperatures are recorded using LabView. Thermocouples are located inside the lauric acid (T2 to T7) as well as on the inlet and outlet (T1 and T8), as seen in Fig. 3a. A pulse counter flowmeter from Omega is connected to a counter/pulse generation module (NI9435) on the DAQ system and read by LabView. The container is made of $1 / 4$-inch acrylic plastic and is un-insulated to allow visual study of the system.

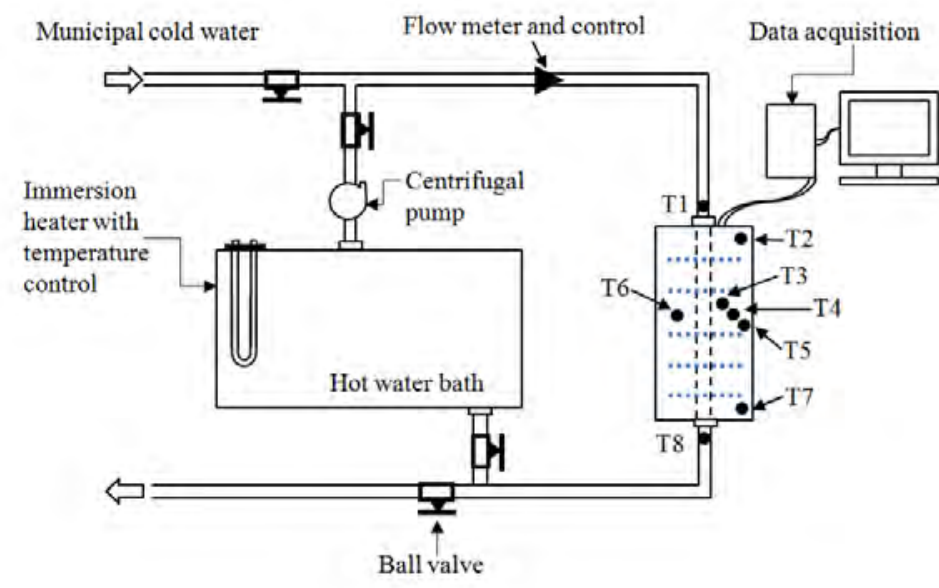

(a)

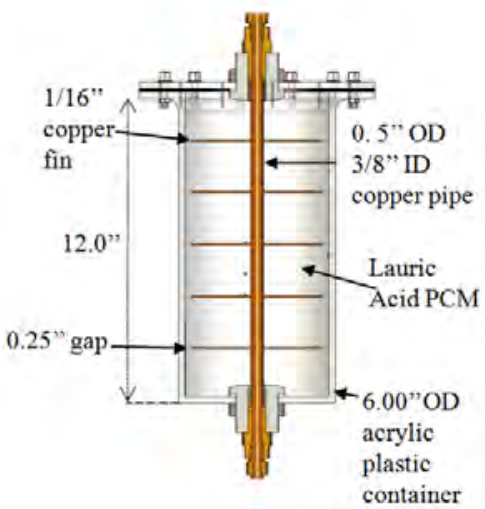

(b)

Fig. 3. (a) Schematic of the experimental setup and (b) PCM container.

The conditions under which the experiments were performed are summarized in Table 2. 
Table 2. Experimental parameters.

\begin{tabular}{lc}
\hline Hot Water Inlet Velocity & $1.5(\mathrm{~m} / \mathrm{s})$ \\
\hline Cold Water Inlet Velocity & $3.5(\mathrm{~m} / \mathrm{s})$ \\
\hline Hot Inlet Temperature & $55 \pm 1\left({ }^{\circ} \mathrm{C}\right)$ \\
\hline Cold Inlet Temperature & $12\left({ }^{\circ} \mathrm{C}\right)$ \\
\hline
\end{tabular}

At the beginning of the experiment, lauric acid was solid in the container at room temperature. Hot water from the constant temperature water bath was pumped through the finned copper pipe, eventually melting the lauric acid. $\mathrm{T}$ he charging portion of the experiment was completed when the system reached steady state. At this point, cold water from the municipal water supply was pumped through the system to solidify the lauric acid and recover the stored thermal energy. The experiment concluded when the lauric acid was at room temperature. The results obtained with this setup were compared to results of numerical simulations.

\section{Numerical Study}

COMSOL Multiphysics (version 4.0a) was used to build a 2D axisymmetric numerical model of the experiment using the Heat Transfer in Solids physics to model the copper and lauric acid, and the Laminar Flow and Heat Transfer in Liquids physics to model the flowing water. The thermophysical properties of water and copper used in the model are those given by COMSOL. For lauric acid, the thermophysical properties used are those presented in Table 1. In this first numerical study, natural convection in the lauric acid was neglected to reduce computing time. An extremely fine mesh was used, with a maximum element size of $2.58 \times 10^{-9} \mathrm{~m}^{2}$. The following boundary and initial conditions were used:

i. Initial temperature of $295 \mathrm{~K}$;

ii. All outside walls have radiation heat losses to the surroundings and natural convection losses are accounted for on the side wall;

iii. No-slip condition on the inner pipe wall;

iv. Inlet temperature and water velocity as in Table 2;

v. No viscous stress and convective flux at the pipe outlet.

Groulx and Ogoh's method of numerically modeling the melting process was used [10]. The simulated time for melting was 11.5 hou rs, and 10 hours for cooling. Simulations took approximately 8 hours to run.

In the experimental setup, two thermocouples (T4 and T6) were placed symmetrically between horizontal fins at the same height but spaced $180^{\circ}$ apart in order to confirm symmetry in the experiment. In the numerical model, a reference point was added to extract information at this location, as shown in Fig 5.

\section{Results and Discussion}

\subsection{Charging Process (Melting)}

Figure 4 presents the temperatures measured experimentally by thermocouples $\mathrm{T} 1$ to $\mathrm{T} 8$ during the charging process. Refer to Fig. 3 for thermocouple positions. 


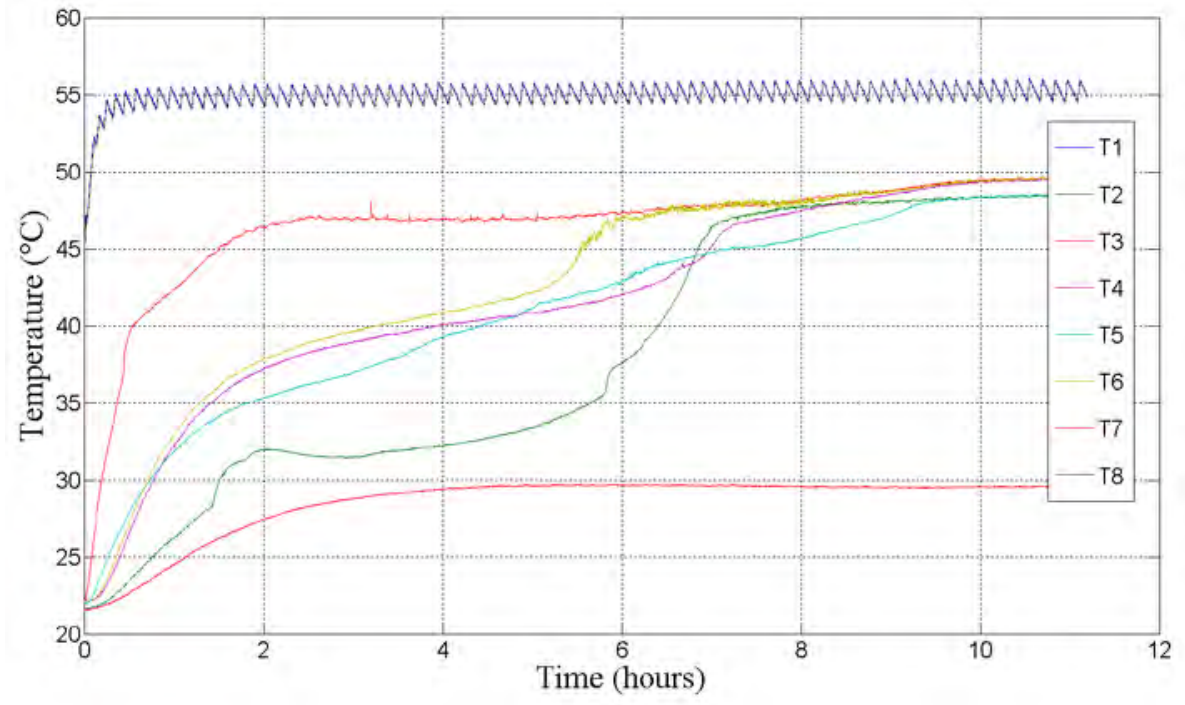

Fig. 4. Experimental temperatures during charging.

The inlet temperature (T1) fluctuates during the charging process due to the emersion heater on/off fluctuations, the outlet temperature (T8) logically follows the same trend. The temperature increases more rapidly close to the pipe and fins (T3), where it takes a longer time for the lauric acid to heat up and melt in regions farther from the pipe, T4 and T6, followed by $\mathrm{T} 5$. The region in the upper corner of the container experiences a fast increase in temperature (T2) after 6 hours, mainly due to the onset of natural convection in this region. The lauric acid does not reach the melting temperature in the bottom corner (T7).

Figure 5 shows the numerically obtained temperatures in the system during charging. The black contour line represents the melting interface.

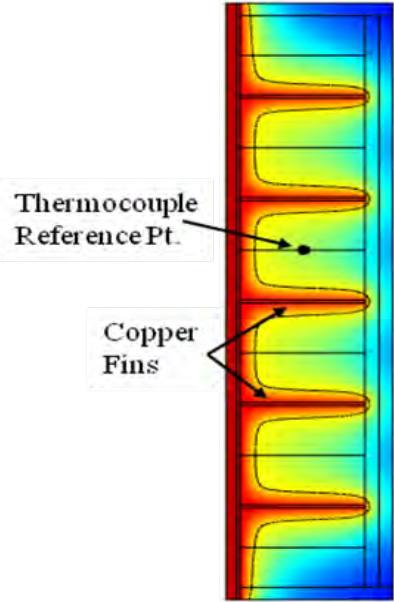

1.5 hours

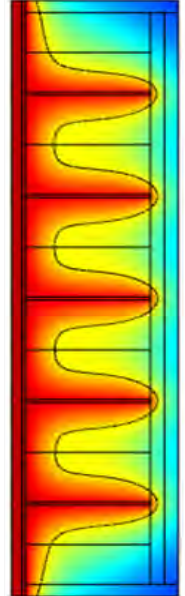

4 hours

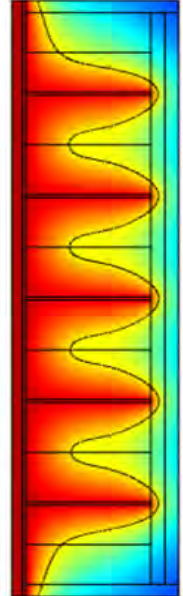

6.5 hours

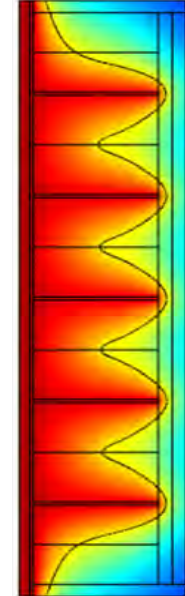

9 hours

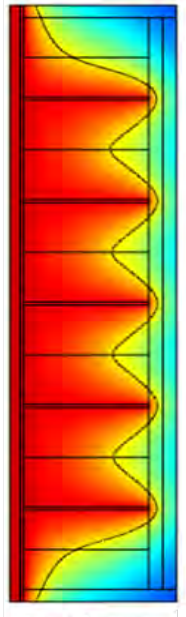

11.5 hours

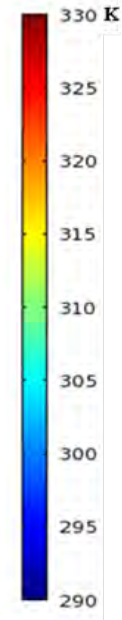

Fig. 5. Numerical temperature plots during charging after 1.5, 4, 6.5, 9 and 11.5 hours.

In Fig. 5, it can be observed that after 1.5 hours the melting process started near the pipe and fin surfaces first, with fairly small temperature increases everywhere else. The heat transfer has been enhanced by adding fins, which is clearly evident by looking at the overall temperature increases far from the pipe. After 11.5 hours of charging, solid lauric acid was still present around the inside wall of the container and in the corners. Experimentally, after 11.5 hours, solid lauric acid was only found in the bottom corners. This difference is thought 
to be due to the effect of natural convection in the system, which increases the overall rate of heat transfer.

The experimentally measured (green and blue lines) and numerically calculated (red line) temperatures during the charging process are presented in Fig. 6.

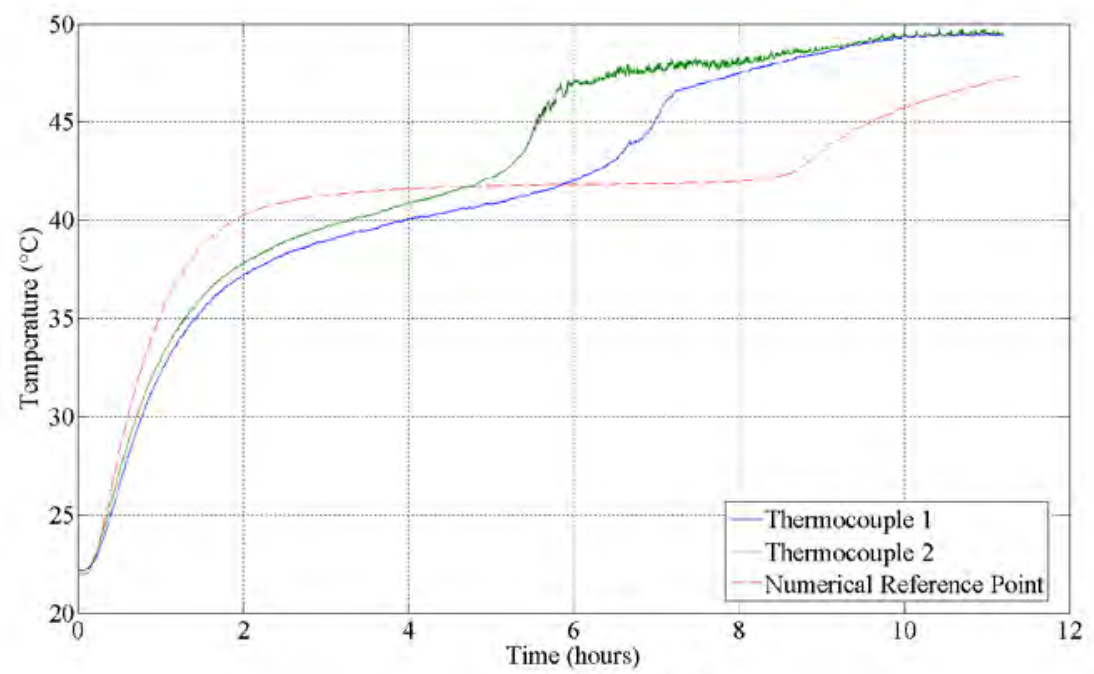

Fig. 6. Numerical validation of melting process in PCM container.

Thermocouples T4 and T6 showed slightly offset temperatures due to asymmetry in the fin assembly which caused one side of the container to heat up faster than the other. Melting occurred between 3 and 8.5 hours in the numerical model. The melting point was reached experimentally after 5 and 6 hours, with fairly constant temperature increases leading to this point; this is consistent with the formation of a mushy region around the thermocouple probes. Complete melting of the lauric acid numerically took longer, possibly due to the lack of natural convection in the model. Experimental results showed a sudden temperature increase in the liquid lauric acid just after melting; this rate of temperature increase in the liquid lauric acid was predicted well by the model.

The numerical model predicted higher effective heat transfer rates initially, leading to a faster temperature increase in the first 2 hours of charging; this is thought to be due to the ideal contact conduction between pipe, fins and lauric acid in the model. In the experimental setup, contact resistances between pipe and fins, as well as between copper surfaces and the solid lauric acid, may have resulted in decreased heat conduction rates at startup.

\subsection{Discharging Process (Solidification)}

Because of the higher flow rate of cold water used during the discharging process, solidification happened over a shorter period of time than melting: 3.5 hours compared to 11 . Figure 7 shows the temperatures in the system during discharging, obtained numerically. The white contour line represents the solidification interface. Solidification of the lauric acid in the numerical model took 4 hours, as seen in Fig. 7.

Figure 8 shows the experimental (blue and green lines) and numerical (red line) cool down temperatures during discharging. 


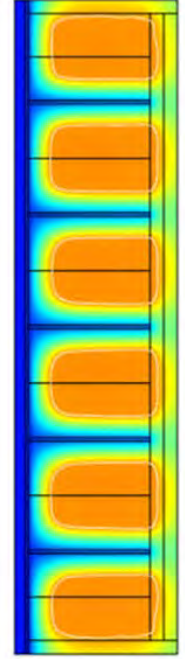

1 hour

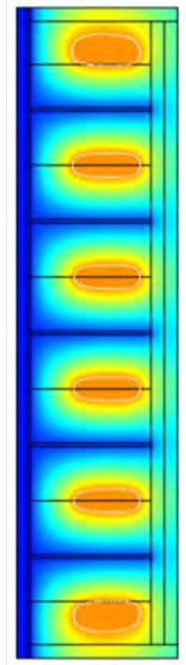

3 hours

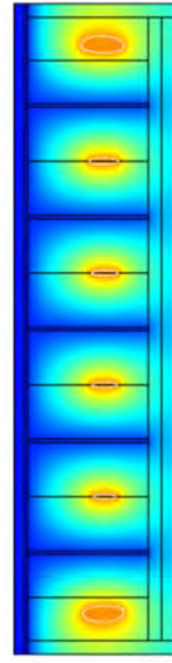

4 hours

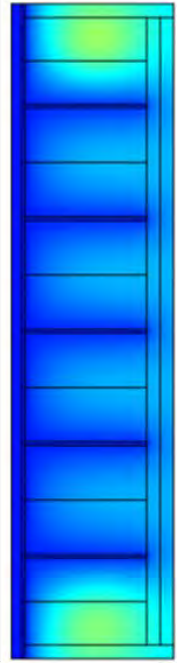

5 hours

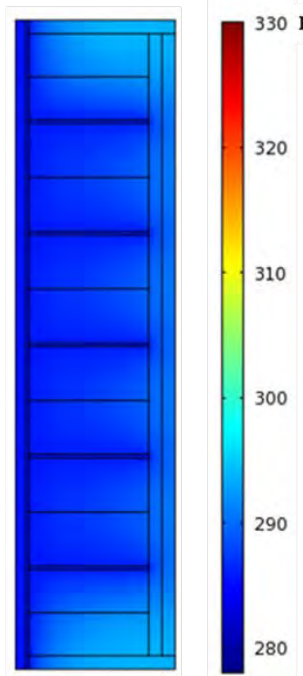

6 hours

Fig. 7. Numerical temperature plots during discharging after 1, 3, 4, 5 and 6 hours.

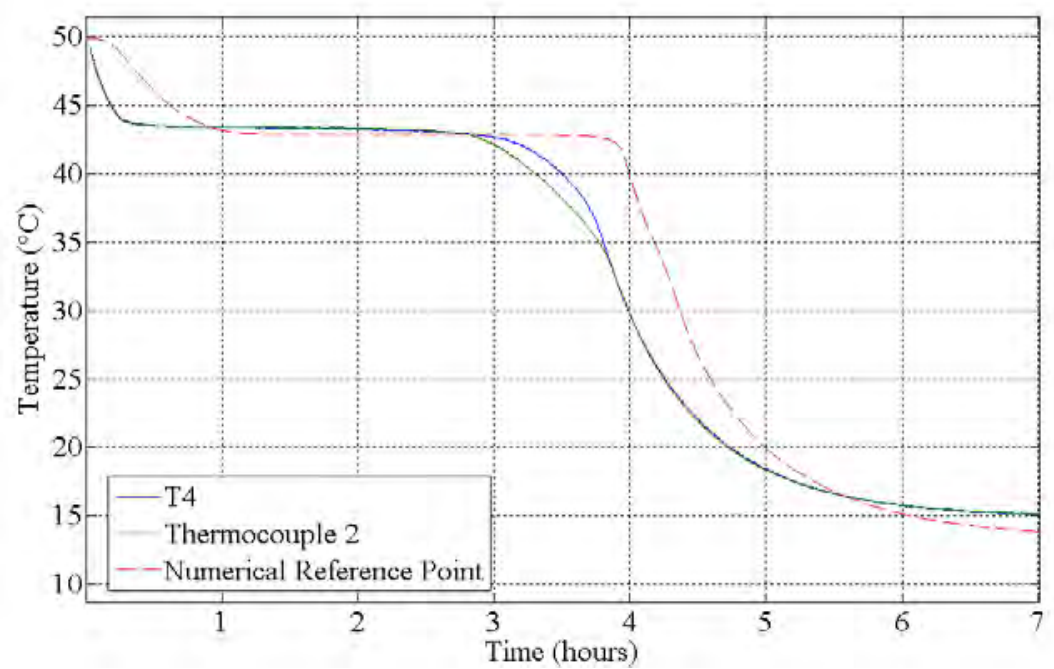

Fig. 8. Numerical validation of solidification process in PCM container.

Of importance to note, the lauric acid solidified at a temperature of $43^{\circ} \mathrm{C}$, different from the predicted DSC measurements, which normally have a certain degree of supercooling. Nucleation happened in the first 15 minutes of cooling during the experiment, due to the presence of the thermocouple probes; this cannot be predicted numerically. However, the model does predict a solidification plateau similar to the one observed experimentally, and only slightly over predicts the time required for solidification at that point in the system.

Throughout the experiment, an insignificant amount of volume change from the solid to liquid phase was observed. Lauric acid did react weakly with copper, taking a bluish color in the liquid state; however this mild reaction does not lead to corrosion in any significant form [11].

\section{Conclusion}

The melting and solidification behavior of lauric acid inside a cylindrical container with a horizontal finned pipe was examined experimentally and numerically. Results for the charging experiment, when compared to the numerical simulations, clearly showed that a mushy region appeared in the system. The presence of natural convection in the liquid melt played a significant role in speeding up the heat transfer and melting process. During 
discharging, the numerical results predicted fairly well the cooling and solidifying behavior observed in the experimental measurements; demonstrating that the effect of natural convection during solidification is for the most part negligible.

\section{Acknowledgments}

We thank Scotian Windfields for their support and expertise, as well as for funding for this project; Eco-Nova Scotia and the Natural Sciences and Engineering Research Council of Canada for their financial support; and the Dalhousie's Mechanical Engineering Department for research space and a flowmeter. The DSC is part of the Facilities for Materials Characterization managed by the Institute for Research in Materials at Dalhousie University.

\section{References}

[1] F. Agyenim, N. Hewitt, P. Eames and M. Smyth, A review of materials, heat transfer and phase change problem formulation for LHTESS, Renewable and Sustainable Energy Reviews 14, 2010, pp.615-628

[2] A.I. Fernandez, M. Martı'nez, M. Segarra, I. Martorell and L.F. Cabeza, Selection of materials with potential in sensible thermal energy storage, Solar Energy Materials \& Solar Cells 94, 2010, pp. 1723-1729

[3] A. Sharma, V.V. Tyagi, C.R. Chen and D. Buddhi, Review on thermal energy storage with $\mathrm{p}$ hase change materials and applications, Renewable and Sustainable Energy Reviews 13, 2009, pp. 318-345

[4] H. El Qarnia, Numerical analysis of a co upled solar collector latent heat storage unit using various phase change materials for heating the water, Energy Conversion and Management 50, 2009, pp. 247-254

[5] A. Sar1, and K. Kaygusuz, Thermal and heat transfer characteristics in a latent heat storage system using lauric acid, Energy Conversion and Management 43, 2002, pp . 2493-2507

[6] A. Shukla, D. Buddhi and R.L. Sawhney, Solar water heaters with phase change material thermal energy storage medium: A review, Renewable and Sustainable Energy Reviews 13, 2009, pp. 2119-2125

[7] Sandnes, B., and Rekstad, J., Supercooling Salt Hydrates: stored enthalpy as a function of temperature, Solar Energy 80, 2006, pp. 616-625

[8] Chemical Properties Handbook, edited by C. L. Yaws, McGraw-Hill, 1999

[9] A11672 - Dodecanoic Acid, Material Safety Data Sheet, Alfa Aesar, 2009, www.alfa.com/en

[10]D. Groulx and W. Ogoh, Thermal behavior of phase change material during charging inside a finned cylindrical latent heat energy storage system: Effects of the arrangement and number of fins, Proceedings of the International Heat Transfer Conference, Washington, DC, USA, 2010

[11]A. Sari and K. Kaygusuz, Some fatty acids used for latent heat storage: thermal stability and corrosion of metals with respect to thermal cycling, Renewable Energy 28, 2003, pp.939-948 